\title{
Neoplasia mucinosa intraductal del páncreas
}

\author{
Jean M Butte ${ }^{1}$, Javiera Torres ${ }^{2}$, Andrés 0 'Brien ${ }^{3}$, \\ Nicolás Jarufe ${ }^{1}, 0$ svaldo Llanos $^{1}$.

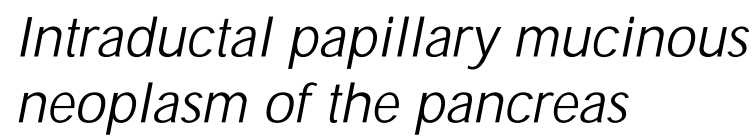

Intraductal papillary mucinous neoplasm of the pancreas is characterized by a dilatation of the main pancreatic duct and/or secondary ducts, mucin production and the absence of ovarian like struma. The symptoms are non-specific and often the diagnosis is incidental. The treatment of choice is surgery, since these tumors may become malignant. The prognosis depends on the type of lesion, whether the excision is complete and lymph node involvement. The aim of this review is to analyze the clinical, diganostic, therapeutic and pathological characteristics of this disease (Rev Méd Chile 2008; 136: 517-27).

(Key words: Carcinoma, pancreatic, ductal; Pancreatic neoplasms)

Recibido el 5 de marzo, 2007. Aceptado el 25 de junio, 2007

Departamentos de Cirugía Digestiva ${ }^{1}$, División de Cirugía, Anatomía Patológica ${ }^{2}$ y Radiología $^{3}$, Facultad de Medicina, Pontificia Universidad Católica de Chile.

$\mathrm{L}$ a Organización Mundial de la Salud ${ }^{1}$ (OMS) definió a la neoplasia mucinosa intraductal del páncreas (NMIP) como un tumor caracterizado por la dilatación del conducto pancreático principal o de sus ramas secundarias, que se encuentra revestido por epitelio cilíndrico, con o sin formación de proyecciones papilares, que se asocia a la producción de mucina y que carece de estroma de tipo ovárico. Esto último permite diferenciarlos de la neoplasia mucinosa quística del páncreas ${ }^{2}$.

Fue descrita por primera vez en 1982 por Ohashi y cols ${ }^{3}$, luego de observar la asociación entre un tumor pancreático y la salida de mucina a través de la ampolla de Vater. Desde entonces,

$\overline{\text { Correspondencia a: Dr. Jean Michel Butte B. Departamento }}$ de Cirugía Digestiva, División de Cirugía, Facultad de Medicina, Pontificia Universidad Católica de Chile. Marcoleta 367. Santiago, Chile. Teléfonos: 56-2-3543462, 56-23543870. Fax 56-2-6329620. E mail: mbutte@gmail.com esta enfermedad ha recibido diferentes nombres, como adenoma velloso, neoplasia papilar, carcinoma papilar, ectasia ductal mucinosa o tumor productor de mucina del páncreas ${ }^{4-6}$.

No existe un cuadro clínico característico y la mayoría de los enfermos consultan por síntomas inespecíficos ${ }^{7,8}$. Por esto, el diagnóstico habitualmente se realiza en forma incidental con estudios imagenológicos del abdomen? .

El tratamiento, en general, es quirúrgico ${ }^{10-12}$. Esta conducta se basa en diferentes estudios que muestran una progresión desde adenoma a adenocarcinoma. La operación dependerá de la localización y extensión de la enfermedad ${ }^{13}$.

El pronóstico de los pacientes depende fundamentalmente de la naturaleza de la lesión, siendo peor en los tumores invasores ${ }^{14}$. En éstos, la sobrevida de los enfermos resecados es mejor que aquellos que presentan un tumor irresecable ${ }^{15}$. También los pacientes con un tumor maligno derivado de una NMIP tienen mejor pronóstico que los con un adenocarcinoma pancreático clásico ${ }^{16}$. 
El objetivo de este trabajo es revisar las características clínicas, imagenológicas, terapéuticas y anatomopatológicas de la NMIP.

Estudio anatomopatológico. La NMIP es una neoplasia productora de mucina que se localiza en el conducto pancreático principal o en sus ramas y se caracteriza por la proliferación intraductal de células mucinosas neoplásicas, las que frecuentemente forman papilas y llevan a la dilatación quística del conducto afectado ${ }^{13,17}$ (Figura 1). La apariencia macroscópica dependerá del conducto pancreático afectado (principal o secundario), el tamaño y la cantidad de mucina producida ${ }^{18}$.

Estos tumores se pueden localizar en cualquier parte del páncreas, pero son más frecuentes en la cabeza (70\%). En 20\% de los enfermos el compromiso es únicamente de la cola y en $10 \%$ es difuso ${ }^{13}$.

En el estudio microscópico se puede observar que la proliferación intraductal puede variar desde un epitelio mucinoso plano que tapiza un conducto dilatado, hasta una compleja formación de papilas ${ }^{17}$.

El principal diagnóstico diferencial es la neoplasia mucinosa quística del páncreas, de la que se diferencia porque carece de estroma de tipo ovárico, existe comunicación de la dilatación quística con los conductos del páncreas, no presenta pseudocápsula y puede existir compromiso multifocal ${ }^{19}$. Esta distinción es muy importante ya que constituye un factor pronóstico ${ }^{13}$.
Dependiendo de las características de las células que revisten los conductos, las NMIP se pueden dividir en ${ }^{20}$ : NMIP de tipo gástrico: Las células que revisten las papilas o la dilatación quística son cilindricas altas, con abundante mucina apical, semejante a las células foveolares gástricas. NMIP de tipo intestinal: Posee papilas largas, villiformes, paralelas, con células que contienen menor cantidad de mucina, pseudoestratificadas y de núcleos alargados, a veces con células caliciformes. NMIP de tipo pancreatobiliar: Con papilas complejas revestidas por células cuboideas, de núcleos redondos, que recuerdan las neoplasias papilares de los conductos biliares.

Es importante destacar que en una NMIP puede haber una mezcla de estos tres tipos.

Existen además otros dos tipos de neoplasia intraductal pancreática que representan variantes de la NMIP: la neoplasia papilar intraductal oncocítica (NPIO) y la neoplasia intraductal tubular $(\mathrm{NIT})^{21}$.

En el año 2000, la OMS $^{2}$ clasificó a las NMIP de acuerdo al grado de atipía epitelial presente en: Adenoma mucinoso papilar intraductal: Si no existe atipia epitelial o es leve (Figura 2A). Neoplasia mucinosa papilar intraductal borderline: Una NMIP con displasia epitelial moderada (Figura 2B). Carcinoma mucinoso intraductal papilar: NMIP con acentuada atipia citoarquitectural (Figura 2C).

Cuando está presente un carcinoma invasor, la OMS recomienda denominarlo como carcinoma

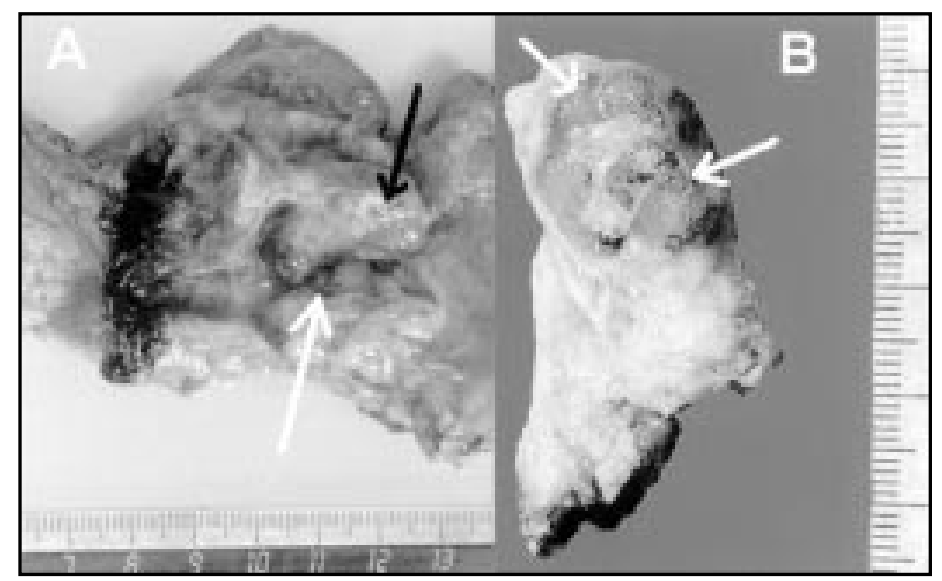

Figura 1. Estudio macroscópico de NMIP. A-NMIP del conducto pancreático principal (flecha blanca) y de conductos secundarios (flecha negra). En el interior de los conductos secundarios se observa mucina. B-NMIP de conductos pancreáticos secundarios (flechas blancas). Se observa mucina en los conductos pancreáticos. 


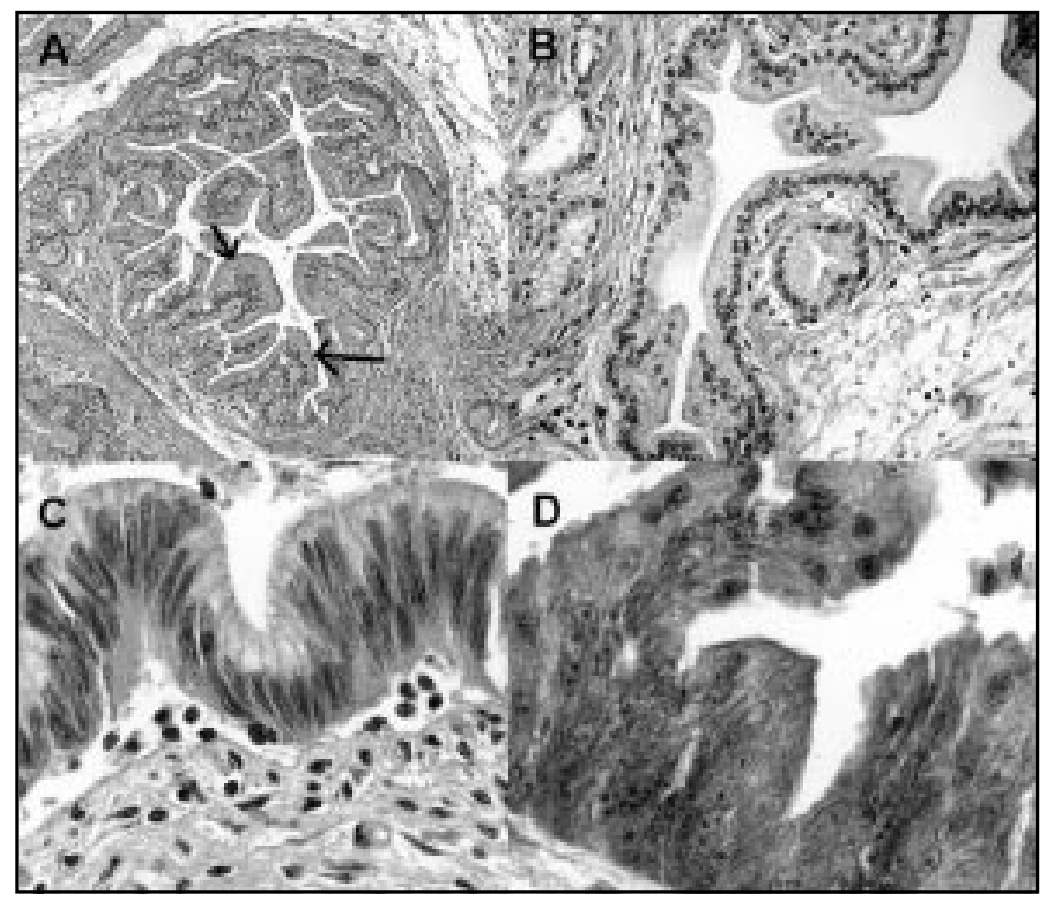

Figura 2. Estudio microscópico de NMIP. A-Proliferación intraductal de células mucinosas neoplásicas con formación de papilas (flechas negras). Tinción H-E 100x. B-Adenoma mucinoso papilar intraductal. Tinción HE 100x. C-NMIP con displasia moderada (borderline). Tinción H-E 400x. D-NMIP con displasia intensa (carcinoma mucinoso intraductal papilar). Tinción H-E 400x.

mucinoso papilar ${ }^{2}$. El carcinoma invasor puede estar presente en 35\% de las NMIP, es más común que se asocie con una NMIP del conducto principal y habitualmente coexiste con displasia de alto grado ${ }^{13}$.

El grupo de expertos del Instituto de Anatomía Patológica de las Fuerzas Armadas de Estados Unidos de Norteamérica en su $4^{\circ}$ fascículo sugieren otra clasificación ${ }^{22}$. Ellos proponen designarlas como NMIP acompañadas del grado de displasia correspondiente (bajo grado o leve, moderada, alto grado o intensa). En caso de existir un foco de carcinoma invasor recomiendan designarlo en forma separada, especificando el tipo histológico, grado y estadío. (Ejemplo: neoplasia mucinosa papilar intraductal del páncreas con carcinoma invasor) ${ }^{22}$.

Citología, estudio intraoperatorio y procesamiento de la pieza para estudio. El diagnóstico diferencial de NMIP y neoplasia mucinosa quística por punción y aspiración con aguja fina es difícil. En ambos casos se identifican células cilíndricas del epitelio ductal con abundante mucina, y dependiendo del grado de displasia presente puede haber mayor o menor atipia citológica. La presencia de estructuras papilares podría orientar hacia una posible NMIP pero el diagnóstico no es definitivo $^{21}$. Por esta razón, la real utilidad de la punción y aspiración con aguja fina en las lesiones quísticas del páncreas es la diferenciación de las lesiones mucinosas de otro tipo de lesiones y la estimación del grado de displasia presente ${ }^{21}$. De todas formas, el diagnóstico requiere del estudio de la pieza operatoria completa ${ }^{21}$.

El estudio intraoperatorio de las lesiones quísticas del páncreas está destinado principalmente al examen del borde quirúrgico en el páncreas y al estudio de lesiones macroscópicas sospechosas de carcinoma invasor, pero no permite un diagnóstico definitivo de NMIP ni descarta la presencia de carcinoma, datos obtenidos sólo con el estudio completo de la pieza ${ }^{21}$. 
El examen histológico de la pieza operatoria debe ser acucioso ya que pueden existir pequeños focos de carcinoma invasor no visibles en el examen macroscópico. Es recomendable que si la pieza no es de gran tamaño se realice un estudio histológico completo de la misma. Si por el contrario, se trata de una pieza grande (ejemplo: $30 \mathrm{~cm}$ ) y no es posible realizar el estudio completo de la misma debe enviarse a estudio el máximo de cortes posibles ${ }^{21}$.

Presentación clínica. La mayoría de las veces se presenta en pacientes mayores (promedio de edad 63 años) y afecta en forma más frecuente a los hombres (relación H:M/3:2) 7,23,24.

Los pacientes con NMIP no tienen un cuadro clínico característico ${ }^{23}$. La mayoría presenta dolor abdominal inespecífico que se puede asociar a episodios de pancreatitis aguda o manifestaciones similares a las de una pancreatitis crónica. Antes de que esta enfermedad estuviera completamente caracterizada, muchos enfermos fueron tratados con el diagnóstico de pancreatitis crónica porque el dolor abdominal recurrente se asociaba al hallazgo de dilatación del conducto pancreático principal en el estudio de imágenes. En los últimos años se ha observado que los enfermos con una NMIP son de mayor edad que los con una pancreatitis crónica ${ }^{12}$.

En un estudio realizado por D'Angelica y cols ${ }^{23}$ a 63 enfermos se observó que 81\% de los pacientes presentaron síntomas. Los más frecuentes fueron dolor abdominal (50\%), pérdida de peso (43\%) y diarrea o esteatorrea (22\%). Se ha especulado que la baja de peso y la esteatorrea estarían asociadas a diferentes niveles de insuficiencia pancreática y que podrían ser secundarias a un aumento en la producción de mucina con obstrucción del conducto pancreático. La ictericia es infrecuente en las lesiones benignas y su presencia debe hacer sospechar la presencia de un tumor maligno. Otras características que sugieren un cáncer asociado a una NMIP son la edad mayor y el diagnóstico tardío o la descompensación a repetición de una diabetes mellitus. Es importante señalar que la ausencia de síntomas no asegura la benignidad de la lesión y se ha observado cáncer in situ o invasor hasta en 30\% de los enfermos asintomáticos ${ }^{13,23}$.

\section{Diagnóstico}

Estudio radiológico. La correcta interpretación de las imágenes en un estudio radiológico permitirá identificar y caracterizar la lesión y facilitará la resección quirúrgica ${ }^{13}$.

El examen de elección para evaluar estas lesiones es la colangiopancreatografía endoscópica retrógrada ${ }^{25}$. En este estudio se pueden observar las características de la papila de Vater (aumento o protrusión, salida de mucina a través de ella) y del conducto pancreático como comunicación de la lesión con ellos, dilatación del conducto principal o de los secundarios (segmentaria o generalizada) y características de la pared del conducto (crecimiento de papilomas o áreas de degeneración maligna) ${ }^{26}$. Sin embargo, es un examen invasivo y no está exento de riesgo. Por esta razón, actualmente se prefiere utilizar otras modalidades de imagen como ultrasonido (US), tomografía computada (TAC) o resonancia magnética $(\mathrm{RM})^{13}$.

NMIP variedad conducto principal. El compromiso puede ser segmentario o difuso. En los diferentes estudios (US-TAC-RM) se puede observar dilatación moderada o acentuada del conducto pancreático principal (Figura 3) y protrusión de la papila hacia el lumen duodenal, signo muy específico de NMIP y que se puede observar en la TAC o en la $\mathrm{RM}^{27}$. Además se pueden identificar imágenes densas en el lumen o en la pared del conducto, que podrían indicar la presencia de un tumor o de acúmulos de mucina. El tumor propiamente tal puede ser difícil de visualizar debido a su pequeño tamaño y a su forma (en general se observa plano).

NMIP variedad conductos secundarios. La mayoría de las veces compromete el proceso uncinado y en la minoría el cuerpo y cola. Se caracteriza por la presencia de múltiples quistes de 1 a $2 \mathrm{~cm}$ de diámetro, interconectados y que contienen mucina o nódulos murales. Se puede asociar a dilatación del conducto principal que en general es secundaria a obstrucción por mucina más densa. En el estudio de imagen se observa dilatación quística de ramas ductales, de contornos lobulados, con múltiples septos y que es hipo O anecogénica al US, hipodensa a la TAC (Figura 4A y B) e hiperintensa en secuencias ponderadas en T2 a la RM (Figura 5A) 28 . 


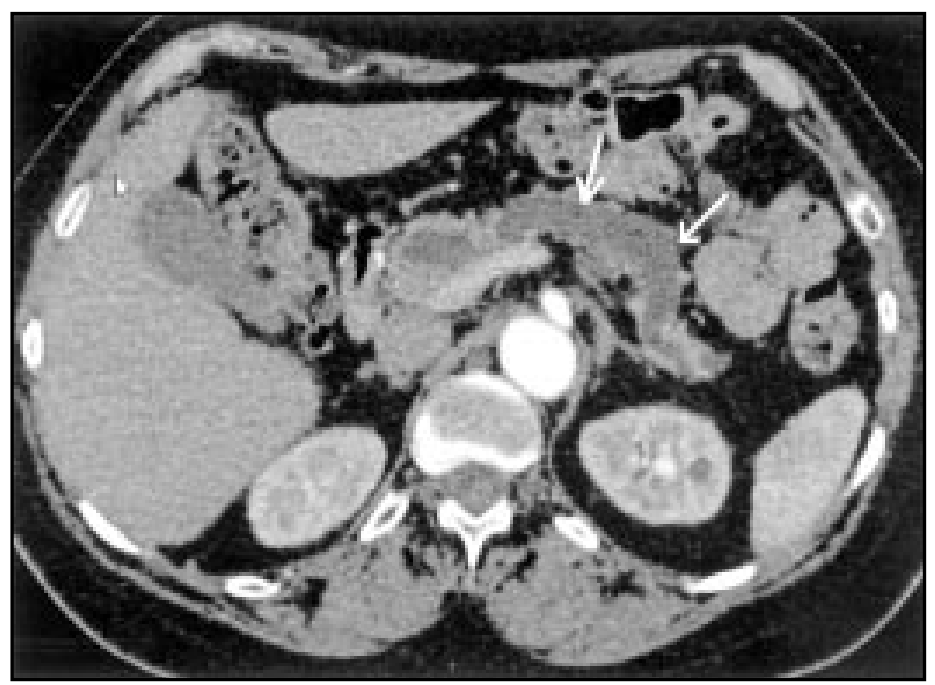

Figura 3. NMIP variedad conducto principal. Corte axial de TAC con múltiples detectores, muestra dilatación difusa del conducto pancreático principal (flechas blancas).

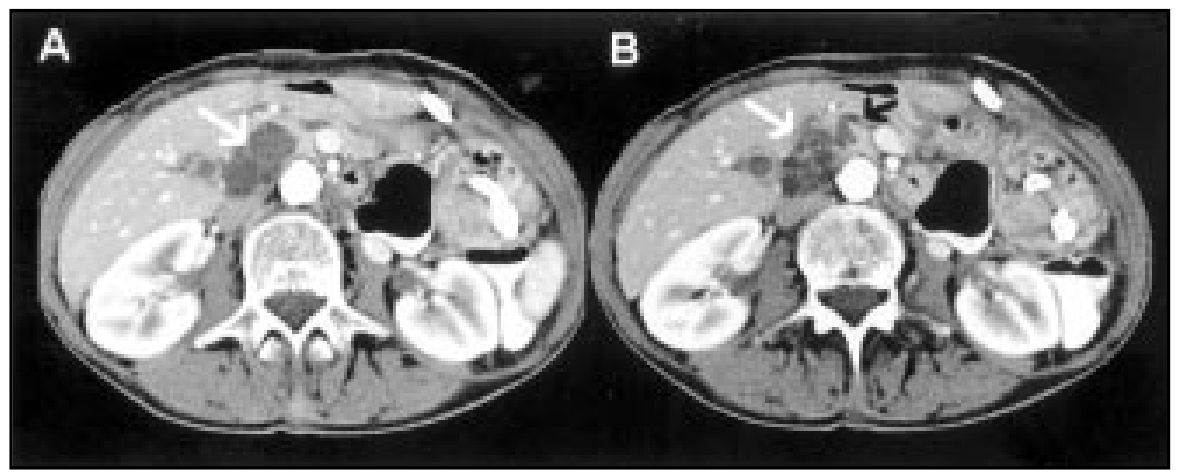

Figura 4 A y B. NMIP variedad conductos secundarios. Cortes axiales de TAC con múltiples detectores. Se observa una lesión multiquística en la cabeza pancreática (flecha blanca) que conecta con el conducto principal (flecha negra).

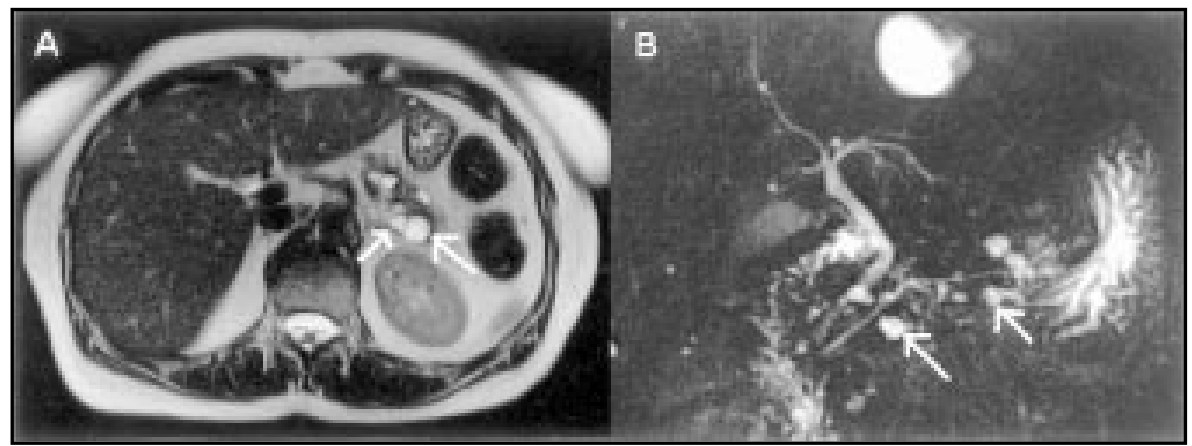

Figura 5. NMIP variedad conductos secundarios (flecha blanca). A-Corte axial de RM en secuencia ponderada en T2 muestra múltiples lesiones quisticas en cola pancreática. B-Colangiopancreatografía por RM. Se observan múltiples lesiones quísticas en forma difusa en el parénquima pancreático sin asociarse a dilatación del conducto principal. 
El desarrollo de la TAC con múltiples detectores y de la $\mathrm{RM}$ (Figura 5B y 6A, B, C), con alta resolución espacial y de contraste respectivamente, ha favorecido el diagnóstico de estas lesiones en forma no invasiva. En estos exámenes se puede demostrar la comunicación de la lesión con el conducto pancreático y la presencia de nódulos murales. El refuerzo de estos últimos sugiere en forma importante una NMIP.

La presencia de compromiso o dilatación acentuada del conducto pancreático principal, compromiso difuso o multifocal, presencia de una masa sólida, de un gran nódulo mural o la obstrucción del colédoco son elementos sugerentes de malignidad ${ }^{29}$.

\section{Otros estudios}

a) Endosonografía: Es un buen estudio para la evaluación de tumores quísticos del páncreas. Se puede obtener imágenes detalladas de la pared y de la arquitectura interna de la lesión. Sin embargo, se ha observado limitaciones por la baja sensibilidad y especificidad en la diferenciación entre los diferentes tipos de tumores pancreáticos y entre la naturaleza benigna o maligna de estos. Puede ser útil para guiar punciones para estudio. La mayor utilidad se ha observado en el segui- miento de las lesiones al facilitar el estudio del páncreas remanente ${ }^{12,30}$.

b) Pancreatoscopia y ultrasonografía intraductal: Es un nuevo método propuesto para la evaluación de las enfermedades pancreáticas, fundamentalmente cuando una estenosis del conducto pancreático no se puede diferenciar de una neoplasia. También puede ser útil en la detección de un carcinoma in situ o de tumores pequeños. La combinación de estas dos técnicas se asocia a un aumento en la sensibilidad diagnóstica que puede llegar a 90\%12,31.

c) Aspiración percutánea con aguja fina: El análisis citopatológico o anatomopatológico del tejido obtenido a través de una aspiración percutánea guiada por endosonografía tiene una alta sensibilidad (91\%) en el diagnóstico de una NMIP cuando están presentes nódulos murales, alteración de los conductos pancreáticos o estenosis del conducto pancreático principal. Es útil cuando no hay eliminación de mucus a través de la papila ${ }^{13}$. En un estudio de Maire y $\operatorname{cols}^{32}$, con esta técnica se logró el diagnóstico en 83\% de los enfermos que no presentaban eliminación de mucus a través de la papila. Sin embargo, la relativa baja celularidad del aspirado es la limitante más importante en el estudio y puede disminuir la sensibilidad a 20\%. En

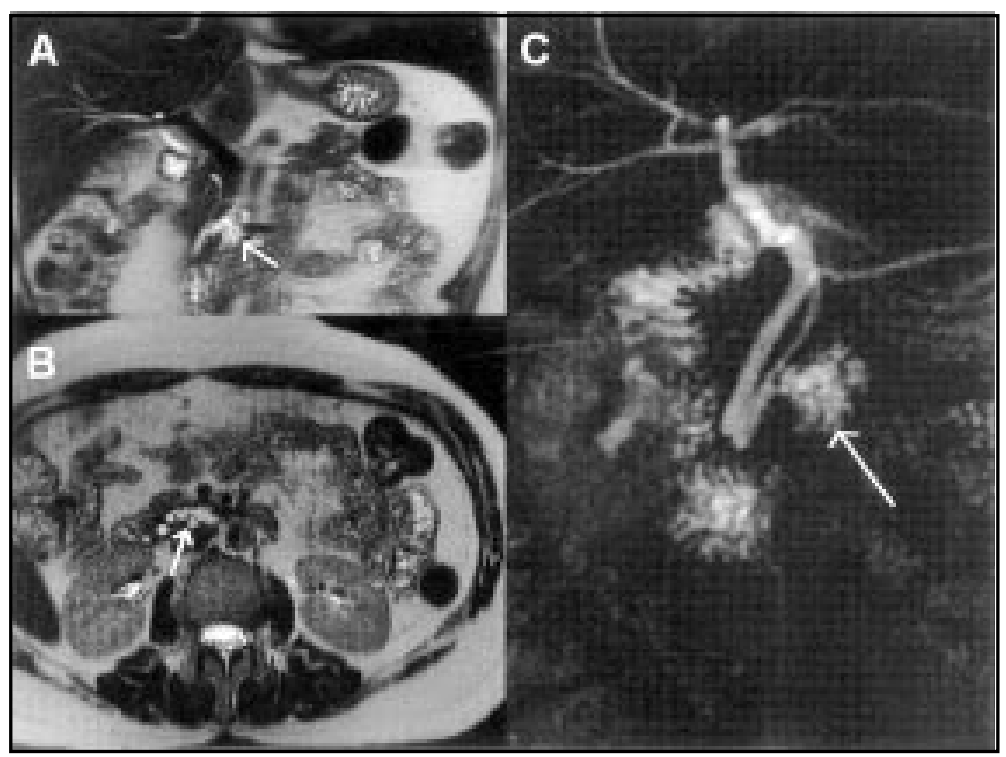

Figura 6. NMIP variedad conductos secundarios. Resonancia magnética de abdomen. Corte coronal (A), axial (B) y colangiorresonancia por RM (C). Imagen ponderada en T2, donde se identifican lesiones quísticas multiloculares en el proceso uncinado que conectan con el conducto pancreático principal (flecha blanca). 
general, la sensibilidad para diferenciar una lesión benigna de una maligna con el estudio del líquido pancreático es de 13\% a 47\%. Además, es importante tener en cuenta que este tipo de estudios entrega información de regiones del conducto pancreático, que pueden no representar la anatomía de todo el conducto y que la mayoría de las veces, la diferenciación entre lesiones benignas y malignas no se podrá obtener sólo con el análisis citológico. Por otra parte, no hay que desconocer que este procedimiento también puede presentar complicaciones como pancreatitis, sangrado, fístulas pancreáticas e implante neoplásico en el trayecto de la punción $^{12}$.

Diagnóstico diferencial. Se realiza principalmente con los cistoadenomas (seroso y mucinoso), cistoadenocarcinomas y los pseudoquistes del páncreas. Los cistoadenomas mucinosos y los cistoadenocarcinomas mucinosos en general se presentan en mujeres de edad media, no están comunicados con el conducto pancreático principal y se ubican con más frecuencia en la cola, donde se identifican quistes mayores de $2 \mathrm{~cm}$. Los cistoadenomas serosos están compuestos por múltiples quistes pequeños menores de $2 \mathrm{~cm}$ (Figura 7), son más frecuentes en mujeres mayores, son benignos, en general se ubican en la cabeza o cuerpo y tampoco están comunicados con el conducto principal. Los pseudoquistes son las lesiones quísticas más frecuentes del páncreas, generalmente están conectados con el conducto pancreático principal y se asocian a signos de pancreatitis crónica como dilatación del conducto principal y calcificaciones. A diferencia de NMIP, no presentan nódulos murales, globos de mucina ni protrusión de la papila 33

Asociación con otras neoplasias. Se ha descrito que con frecuencia, las NMIP se pueden asociar a otras neoplasias extrapancreáticas ${ }^{12}$. Sugiyama y $\mathrm{cols}^{34}$ evaluaron a 42 enfermos y encontraron que $48 \%$ de ellos desarrollaron un tumor extrapancreático y que en 36\% éste fue maligno. El mayor compromiso se observó en el colon $(33,3 \%)$ y en el estómago (26,7\%).

En otro estudio, Choi y cols ${ }^{35}$ evaluaron a 61 enfermos con una NMIP y observaron que 24 (39\%) de ellos presentaron uno o más tumores extrapancreáticos, la mayoría malignos. El estómago y el colon también fueron los órganos que con más frecuencia se comprometieron. Se diagnosticaron 8 tumores antes de la NMIP, 15 en forma simultánea y 3 en forma posterior. Estos enfermos se caracteriza-

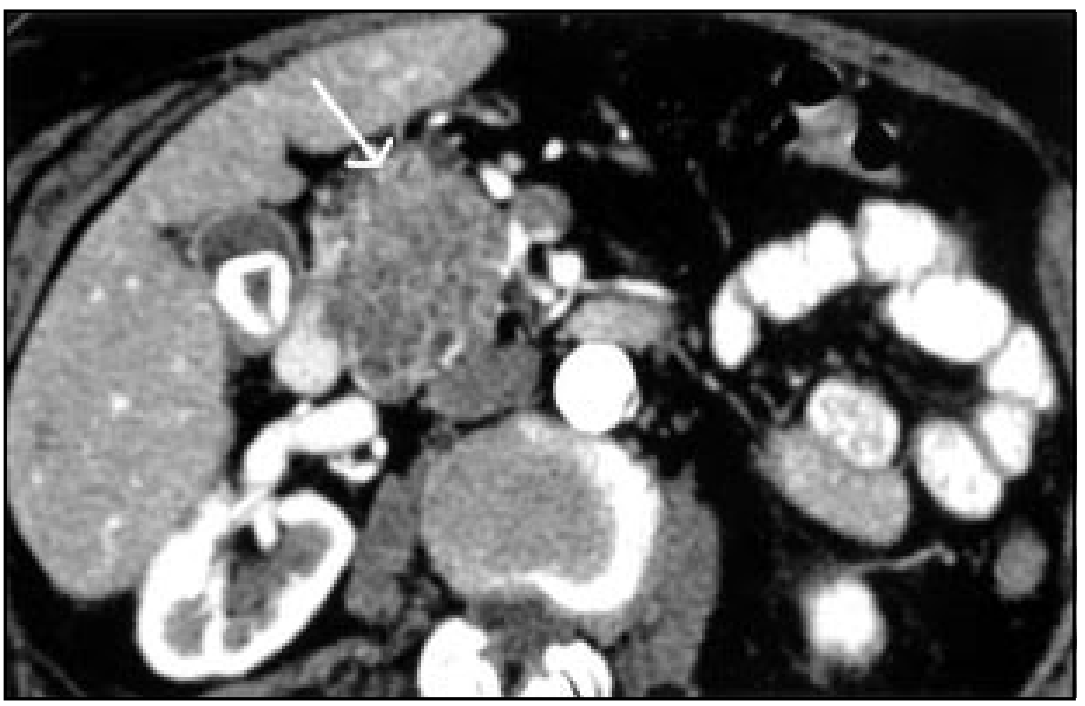

Figura 7. Cistoadenoma seroso. Corte axial de TAC con múltiples detectores muestra una lesión multiquística en la cabeza pancreática sin conexión con el conducto principal (flecha blanca). Los quistes son menores a $2 \mathrm{~cm}$. 
ron por presentar un mayor porcentaje de NMIP borderline y de ubicación distal. Sin embargo, no se observaron diferencias significativas en la sobrevida de los pacientes con una NMIP asociada o no con un tumor extrapancreático. Otro hallazgo importante fue que los enfermos con una NMIP tuvieron un significativo mayor porcentaje de tumores extrapancreáticos que los enfermos con un tumor quístico mucinoso o con un adenocarcinoma del páncreas.

Los cambios genéticos pueden tener un rol importante en el desarrollo de tumores extrapancreáticos, especialmente en los cánceres del tracto digestivo. La carcinogénesis de las NMIP y del cáncer colorrectal es similar, desde la presencia de un adenoma hasta el desarrollo de un cáncer invasor y ambas tienen alteraciones comunes como mutaciones en el oncogen k-ras ${ }^{36}$, la sobreexpresión del gen HER-2/neu ${ }^{37}$ y la inactivación del gen STK11/ LKB1 $^{38}$. Todos los enfermos con una NMIP deberían ser estudiados con una endoscopia digestiva alta y una colonoscopia, con el fin de detectar en forma precoz un tumor gástrico o colónico asociado.

Tratamiento. Tratamiento quirúrgico: El principal objetivo de la cirugía es realizar una completa resección de la enfermedad, con márgenes negativos, con el fin de disminuir la posibilidad de recurrencia en el páncreas remanente ${ }^{12,13,24}$. El tratamiento quirúrgico depende fundamentalmente del estado clínico del enfermo al momento de la cirugía y de las características de la lesión en el estudio de imágenes.

- Tumores del conducto principal: La frecuencia de malignidad (cáncer in situ o invasor) es de $60 \%$ a $92 \%$ y aproximadamente dos tercios de estas neoplasias son invasoras al momento del diagnóstico $^{13}$. Se ha observado que los enfermos sintomáticos, que presentan un conducto mayor de $15 \mathrm{~mm}$ o tienen nódulos parietales tienen una mayor probabilidad de presentar un tumor invasor. En un estudio de Salvia y cols ${ }^{8}$ se observó que las neoplasias malignas las presentaban los pacientes de mayor edad, con ictericia y con descompensación de la diabetes. Sin embargo, también se observó que $29 \%$ de los enfermos con cáncer fueron asintomáticos y por lo tanto, el análisis clínico y radiológico no permitió determinar con exactitud el carácter de la lesión.

La sobrevida a 5 años de los enfermos con lesiones benignas o no invasivas es cercana a $100 \%$, mientras que en los enfermos con lesiones malignas es de $36 \%-60 \% 13$, por lo que se sugiere que la resección al momento del diagnóstico debe ser el tratamiento de elección de los enfermos con una NMIP que comprometa el conducto principal.

- Tumores de conductos secundarios: La posibilidad de malignidad es de $6 \%$ a $46 \%$ y de cáncer invasor de $0 \%$ a $31 \%{ }^{13}$. Esto se asocia fundamentalmente con el tamaño del conducto $(>30 \mathrm{~mm}$ ) y con la presencia de nódulos parietales. De esta forma, aquellos enfermos asintomáticos, con dilatación del conducto periférico hasta $30 \mathrm{~mm}$ y sin nódulos parietales podrían ser observados y sometidos a seguimiento estricto con imágenes. Es importante considerar que esta conducta debe ser evaluada para cada caso en particular. En los enfermos sintomáticos, que requieran un seguimiento prolongado o que exista duda diagnóstica, la cirugía será el tratamiento de elección ${ }^{13}$.

El tratamiento quirúrgico de elección en los enfermos con sospecha de malignidad es la pancreatectomía (pancreatoduodenectomía, pancreatectomía corporocaudal o pancreatectomía total) con disección linfática ${ }^{12,13}$. En los pacientes que no presentan criterios de malignidad (clínicos, de laboratorio o imagenológicos), se puede plantear una resección segmentaria, pero teniendo en cuenta la morbilidad asociada a esta intervención y la posibilidad de una futura resección radical.

Tratamiento adyuvante: No existe un rol demostrado de las terapias adyuvantes en los enfermos con una NMIP ${ }^{12}$.

\section{Pronóstico}

El pronóstico de los enfermos está dado por la resección quirúrgica completa y por la ausencia de un carcinoma invasor ${ }^{12,13,23,24}$. Los casos de NMIP que han presentado metástasis posterior a la resección, se han asociado a la presencia de focos de carcinoma invasor no detectados, por inadecuado muestreo de la pieza operatoria o por la no inclusión de estos focos en la resección. También se han descrito casos de recurrencia con márgenes de resección negativos, que se explicarían por la multicentricidad que caracteriza a estas neoplasias. La presencia de carcinoma invasor afecta en forma adversa el pronóstico; el carcinoma tubular parece ser casi tan agresivo como el adenocarcinoma ductal convencional no asociado 
a NMIP, pero el carcinoma mucinoso parece tener un pronóstico relativamente mejor, con una sobrevida de $55 \%$ a 5 años, incluso en aquellos pacientes con tumores grandes y metástasis en ganglios linfáticos.

Factores pronósticos. Se han estudiado diferentes factores pronósticos como:

- a) Enfermedad maligna: En la mayoría de las oportunidades no se podrá diferenciar una NMIP maligna de una benigna en el estudio preoperatorio. Aun en los enfermos con enfermedad catalogada como benigna es posible la recurrencia y en estos casos, se deberá pensar como primera posibilidad, que un componente maligno no apreciado en la cirugía original o un foco sincrónico debido a multicentricidad de la enfermedad estuvo presente. Se ha observado que las NMIP benignas tienen un mejor pronóstico que las malignas y es el principal factor asociado con la sobrevida de estos enfermos. En estos casos, la recurrencia en el tejido pancreático o extrapancreático se puede observar en $50 \%$ a $90 \%$ de los enfermos, disminuyendo la sobrevida a 5 años a menos de 50\%. En el estudio de D'Angelica y $\operatorname{cols}^{23}$ se observó que la sobrevida de los tumores menores de $1,9 \mathrm{~cm}$ fue de $67 \%$, la que descendió a $42 \%$ en los de 2 o más cm. Esto también está relacionado con la mayor posibilidad que existe de diseminación a los ganglios linfáticos. Hay que destacar que en la mayoría de los enfermos el diagnóstico se realiza cuando hay pequeños focos de adenocarcinoma y por esto, los enfermos tienen mejor pronóstico que los con un adenocarcinoma clásico del páncreas ( $45 \%$ vs $15 \%-25 \%$ ). Sin embargo, si se comparan ambos cánceres según etapas, la sobrevida de los enfermos con un cáncer derivado de una NMIP es levemente superior a la de aquellos con un cáncer clásico, lo que sugiere que esta enfermedad también es de mal pronóstico ${ }^{12}$.

b) Metástasis linfonodales: Las NMIP malignas se asocian en bajo porcentaje a compromiso linfonodal y usualmente compromete los ganglios peripancreáticos. Se ha observado compromiso de éstos en $29 \%$ a $46 \%$ de los enfermos con tumores malignos y se asocia a un peor pronóstico, disminuyendo la sobrevida a 5 años de $83 \%$ a 30\%, según lo comunicado en el Memorial Sloan-Kettering 23 . En otro estudio ${ }^{24}$ se reportó una sobrevida a 1, 2 y 5 años de
$45 \%, 24 \%$ y $0 \%$ cuando los ganglios estuvieron comprometidos, comparado con 95\%, 95\% y $85 \%$ cuando no estuvieron comprometidos.

c) Invasión vascular y neural: Se asocia a un peor pronóstico, disminuyendo la sobrevida a 5 años de $83 \%$ y $77 \%$ en los enfermos que no presentan a $0 \%$ y $57 \%$ en los que presentan ${ }^{12}$.

d) Tipo histológico: El tipo coloideo tiene mejor pronóstico que el tubular. Los tumores tubulares se asocian a mayor compromiso ganglionar y a compromiso vascular ${ }^{12}$

e) Bordes quirúrgicos: No hay información concreta en relación al pronóstico de los enfermos según los bordes quirúrgicos. En el estudio de D'Angelica y cols ${ }^{23}$ se observó que el pronóstico estaría determinado fundamentalmente por las características del tumor primario más que por el compromiso del borde quirúrgico, pero Sohn y cols observaron que la sobrevida a 1, 2 y 5 años de los enfermos con bordes negativos fue de $78 \%, 62 \%$ y $46 \%$ a diferencia de los enfermos con bordes positivos en que fue de $40 \%, 0 \%$ y $0 \% 0^{24}$.

f) Compromiso del conducto pancreático principal $\mathrm{v} / \mathrm{s}$ conductos secundarios: Los enfermos con tumores localizados en los conductos secundarios tendrían un mejor pronóstico. En un estudio realizado por Sohn y cols ${ }^{24}$ se observó que la sobrevida a 5 años fue de 69\%, 56\% y $62 \%$ en los enfermos con tumores de conducto secundarios, conducto principal y mixto, pero esta diferencia no fue estadísticamente significativa.

Seguimiento. En los enfermos no operados la opción de seguimiento se apoyará en la edad del enfermo, las enfermedades asociadas y la estimación del riesgo de cáncer de la lesión ${ }^{13}$. El riesgo de cáncer es mayor en las lesiones que comprometen el conducto principal. En aquellos con compromiso de conductos periféricos se ha observado que existe mayor posibilidad de malignización en los pacientes sintomáticos. En pocos trabajos se han estudiado factores de riesgo de malignización en pacientes asintomáticos. Sin embargo, y a pesar de lo limitado de ellos, en el seguimiento entre 12 y 36 meses no se desarrolló un cáncer invasor en los pacientes cuyo conducto principal medía $6 \mathrm{~mm}$ o menos o no presentaba nódulos murales. Idealmente el estudio imagenológico con que se inicia el seguimiento debe proveer una adecuada información en relación al 
tamaño de la lesión, del conducto pancreático principal y la presencia de nódulos parietales. Se sugiere un seguimiento anual en lesiones menores de $10 \mathrm{~mm}$, entre 6 y 12 meses en las de $10-20 \mathrm{~mm}$ y cada 3 a 6 meses en las mayores de $20 \mathrm{~mm}^{13}$.

Si en el seguimiento la lesión se torna sintomática, presenta un tamaño mayor de $3 \mathrm{~cm}$, el conducto pancreático principal mide más de $6 \mathrm{~mm}$ o aparecen nódulos parietales, se deberá indicar la resección. El intervalo de observación se podrá ampliar después de 2 años sin modificaciones ${ }^{13}$.

En los pacientes en que se ha resecado una NMIP benigna, se debería realizar un seguimiento con el fin de detectar un posible compromiso del resto del tejido pancreático. Se estima que $7 \%$ de

\section{REFERENCIAS}

1. Kloppel G, Solcia E, Longnecker D, Capella C, Sobon L. Histological Typing of tumors of the exocrine pancreas. World Health Organization. Berlín, Springer, 1996; 1-61.

2. Longnecker D, Adler G, Hruban R, Kloppel G. Intraductal papillary-mucinous neoplasms of the pancreas. In Hamilton S, Aaltonen L (eds). Classification of tumors, Pathology and genetics of tumors of the Digestive System. World Health Organization. Lyon; IARC Press, 2000; 237-41.

3. Оhнashi K, Murakami $Y$, Takekoshi T. Four cases of mucous secreting pancreatic cancer. Progress in Diagnostic Endoscopy 1982; 20: 348-51.

4. Nakazawa S, Yamao K, Yamada M, Naito Y, Kimoto E, INUI K ET AL. Study of the classification of mucinproducing cystic tumors of the pancreas. Nippon Shokakibyo Gakkai Zasshi 1988; 85: 924-32.

5. Yamada M, KozuKa $S$, Yamao K, Nakazawa S, Naitoh Y, Tsuкамото Y. Mucin-producing tumor of the pancreas. Cancer 1991; 68: 159-68.

6. Yamaguchi K, Ogaiwa Y, Chijina K, Tanaka M. Mucin-hypersecreting tumors of the pancreas: assessing the grade of malignancy preoperatively. Am J Surg 1996; 171: 427-31.

7. Sohn T, Yeo C, Cameron J, Iacobuzio-Donahue C, Hruban R, LILEMoE K. Intraductal papillary mucinous neoplasms of the pancreas: an increasingly recognized clinicopathologic entity. Ann Surg 2001; 234: 313-21.

8. Salvia R, Fernández-del Castillo C, Bassi C, Thayer S, Falconi M, Mantovani W et al. Main-duct los enfermos pueden desarrollar nuevos focos de NMIP en el tejido pancreático remanente, por lo que se sugiere un control anual. Los enfermos con tumores malignos tienen un mayor riesgo de recurrencia y probablemente deben ser evaluados cada 6 meses. Los niveles de CEA o de CA 19-9 no han demostrado ser útiles en el seguimiento ${ }^{13}$.

\section{Agradecimiento}

Los autores agradecen al Dr. Ignacio Duarte G, Profesor Titular del Departamento de Anatomía Patológica de la Pontificia Universidad Católica de Chile, por la lectura crítica y los comentarios sobre el manuscrito.

intraductal papillary mucinous neoplasms of the pancreas: clinical predictors of malignancy and long-term survival following resection. Ann Surg 2004; 239: 678-85.

9. Yamao K, Ohashi K, Nakamura T, Suzuki T, Watanabe Y, SHimizU Y ET AL. Evaluation of various imaging methods in the differential diagnosis of intraductal papillary-mucinous tumor (IPMT) of the pancreas. Hepatogastroenterology 2001; 48: 962-6.

10. TRAVERSO W. Surgical treatment of intraductal papillary mucinous neoplasms of the pancreas: The aggressive approach. J Gastrointest Surg 2002; 6: 662-3.

11. Fernández del Castillo C. Surgical treatment of intraductal papillary mucinous neoplasms of the pancreas: The conservative approach. J Gastrointest Surg 2002; 6: 660-1.

12. Sakorafas G, Sarr M, van de Velde C, Peros G. Intraductal papillary mucinous neoplasms of the pancreas: A surgical perspective. Surg Oncol 2005; 14: 155-78.

13. Tanaka M, Chari S, Adsay V, Fernández del Castillo C, Falconi M, Shimizu M et al. International consensus guidelines for management of intraductal papillary mucinous neoplasm and mucinous cystic neoplasms of the pancreas. Pancreatology 2006; 6: 17-32.

14. Sugiura H, Kondo S, Islam H, Ito K, Ono K, Morikawa $\mathrm{T}$ Eт AL. Clinicopathologic features and outcomes of intraductal papillary-mucinous tumors of the pancreas. Hepatogastroenterology 2002; 49: 263-7.

15. Raimondo M, Tachibana I, Urrutia R, Burgart L, DiMAgno E. Invasive cancer and survival of 
intraductal papillary mucinous tumors of the pancreas. Am J Gastroenterol 2002; 97: 2553-8.

16. Maire F, Hammel P, Terris B, Paye F, Scoazec J, Cellier C ET al. Prognosis of malignant intraductal papillary mucinous tumours of the pancreas after surgical resection. Comparison with pancreatic ductal adenocarcinoma. Gut 2002; 51: 717-22

17. Adsay V. Intraductal papillary mucinous neoplasms of the pancreas: Pathology and molecular genetics. J Gastrointest Surg 2002; 6: 656-9.

18. Kobari M, Egaiwa S, Shibuya K, Shimamura H, Sunamura $\mathrm{M}$, TAKEDA K ET AL. Intraductal papillary mucinous tumors of the pancreas comprise 2 clinical subtypes: Differences in clinical characteristics and surgical management. Arch Surg 1999; 134: 1131-6.

19. Zamboni G, Scarpa A, Bogina G, Iacono C, Bassi C, Talamini G et al. Mucinous cystic tumors of the pancreas: Clinicopathological features, prognosis, and relationship to other mucinous cystic tumors. Am J Surg Pathol 1999; 23: 410-22.

20. Klimstra D. Cystic, mucin-producing neoplasms of the pancreas: the distinguishing feature of mucinous cystic neoplasms and intraductal papillary mucinous neoplasms. Seminars in Diagnostic Pathology 2005; 22: 318-29.

21. Adsay N, Adair C, Heffess C, Kuimstra D. Intraductal oncocytic papillary neoplasms of the pancreas. Am J Surg Pathol 1996; 20: 980-94.

22. Hruban R, Pitman M, Kuimstra D. Tumors of the pancreas. Atlas of tumor pathology, $4^{\text {th }}$ series, fascicle. Washington DC, American Registry of Pathology, 2007.

23. D'Angelica M, Brennan M, Suriawinata A, Kuimstra D, Conton K. Intraductal papillary mucinous neoplasms of the pancreas: an analysis of clinicopathologic features and outcome. Ann Surg 2004; 239: 400-8.

24. Sohn T, Yeo C, Cameron J, Hruban R, Fukushima N, Campbell K, Lillemoe K. Intraductal papillary mucinous neoplasms of the pancreas: an updated experience. Ann Surg 2004; 239: 788-97.

25. Yamaguchi K, Chijiwa K, Shimizu S, Yokohata K, Morisaki T, Tanaka M. Comparison of endoscopic retrograde and magnetic resonance cholangiopancreatography in the surgical diagnosis of pancreatic diseases. Am J Surg 1998; 175: 203-8.

26. Koito K, Namieno T, Ichimura T, Yama N, Hareyama M, Morita K et al. Mucin-producing pancreatic tumors: comparison of MR cholangiopancreatography with endoscopic retrograde cholangiopancreatography. Radiology 1998; 208: 231-7.
27. Lim J, LeE G, OH Y. Radiologic spectrum of intraductal papillary mucinous tumor of the pancreas. Radiographics 2001; 21: 323-40.

28. Procacci C, Megibow A, Carbognin G, Guarise A, Spoto E, Biasiutti C ET AL. Intraductal papillary mucinous tumor of the pancreas: A Pictorial Essay. RadioGraphics 1999; 19: 1447-63.

29. Kawamoto S, Horton K, Lawler L, Hruban R, Fishman E. Intraductal Papillary Mucinous Neoplasm of the Pancreas: Can benign lesions be differentiated from malignant lesions with multidetector CT? RadioGraphics 2005; 25: 1451-70.

30. Song M, Lee S, Kim M, Lee H, Kim K, Kim H et al. EUS in the evaluation of pancreatic cystic lesions. Gastrointest Endosc 2003; 57: 891-6.

31. Hara T, Yamaguchi T, Ishihara T, Tsuyuguchi $T$, Kondo F, Kato K et al. Diagnosis and patient management of intraductal papillary-mucinous tumor of the pancreas by using peroral pancreatoscopy and intraductal ultrasonography. Gastroenterology 2002; 122: 34-43.

32. Maire F, Couvelard A, Hammel P, Ponsot P, Palazzo L, Aubert A et al. Intraductal papillary mucinous tumors of the pancreas: the preoperative value of cytologic and histopathologic diagnosis. Gastrointest Endosc 2003; 58: 701-6.

33. Fukukura N, Fujiyoshi F, Sasaki M, Inoue H, YoneZAWA S, NAKAJO M. Intraductal Papillary mucinous tumors of the pancreas: thin-section helical CT findings. AJR Am J Roentgenol 2000; 174: 441-7.

34. Sugiyama M, Aтомi Y. Extrapancreatic neoplasms occur with unusual frequency in patients with intraductal papillary mucinous tumors of the pancreas. Am J Gastroenterol 1999; 94: 470-3.

35. Chol M, Kim S, Han S, Jang J, Park Y. High incidence of extrapancreatic neoplasms in patients with intraductal papillary mucinous neoplasms. Arch Surg 2006; 141: 51-6.

36. Uemura K, Hiyama E, Murakami Y, Kanehiro T, Ohge H, SuEDA T ET AL. Comparative analysis of K-ras point mutation, telomerase activity, and p53 overexpression in pancreatic tumours. Oncol Rep 2003; 10: 277-83.

37. Terada T, Ohta T, Nakanuma Y. Expression of oncogene products, antioncogene products and oncofetal antigens in intraductal papillary mucinous neoplasms of the pancreas. Histopathology 1996; 29: 355-61.

38. Sato N, Rosty C, Jansen M, Fukushima N, Ueki T, Yeo C ET AL. STK11/LKB1 Peutz-Jeghers gene inactivation in intraductal papillary-mucinous neoplasms of the pancreas. Am J Pathol 2001; 159: 2017-22. 